\title{
SOME ALGEBRAIC AND TOPOLOGICAL RANDOM FIXED POINT THEOREMS WITH APPLICATIONS TO NONLINEAR RANDOM INTEGRAL EQUATIONS
}

\author{
B. C. DHAGE
}

\begin{abstract}
In this paper some algebraic and topological random fixed point theorems are proved involving the three random operators on a Banach algebra and they are further applied to a certain nonlinear functional random integral equation of mixed type for proving the existence as well as existence of extremal random solutions under the generalized Lipschizicity, Carathéodory and monotonicity conditions.
\end{abstract}

\section{Introduction}

Throughout this paper, let $(\Omega, \mathcal{A})$ denote a measurable space, $X$ a separable Banach space with a norm $\|\cdot\|$, and let $\beta_{X}$ denote a $\sigma$-algebra of all Borel subsets of $X$. By $2^{X}, C B(X)$ and $K(X)$ we denote respectively the classes of all non-empty, closed and bounded, and compact subsets of $X$. A function $x: \Omega \rightarrow X$ is called measurable if for any $B \in \beta_{X}$.

$$
x^{-1}(B)=\{\omega \in \Omega \mid x(\omega) \in B\} \in \mathcal{A} .
$$

We recall that a multi-valued mapping $F: \Omega \rightarrow 2^{X}$ is called measurable if for any open subset G of X,

$$
F^{-1}(G)=\{\omega \in \Omega \mid F(\omega) \cap G \neq \phi\} \in \mathcal{A} .
$$

Notice that if $F(\omega) \in K(X)$ for each $\omega \in \Omega$, then $F$ is measurable if and only if $F^{-1}(C) \in$ $\mathcal{A}$ for a closed subset $C$ of $X$. See Himmelberg [15].

A measurable mapping $\xi: \Omega \rightarrow X$ is called measurable selector of the multi-valued mapping $F: \Omega \rightarrow 2^{X}$ if $\xi(\omega) \in F(\omega)$ for all $\omega \in \Omega$. A mapping $T: \Omega \times X \rightarrow X$ is called a random operator if $T(\cdot, x)$ is measurable for all $x \in X$ and it is generally expressed as $T(\omega, x):=T(\omega) x$. A measurable function $\xi: \Omega \rightarrow X$ is called a random fixed point of the random operator $T(\omega): \Omega \times X \rightarrow X$ if $T(\omega, \xi(\omega))=T(\omega) \xi(\omega)=\xi(\omega)$ for all $\omega \in \Omega$.

Let $T: X \rightarrow X$. Then $T$ is called continuous if for any open subset $G$ of $X, T^{-1}(G)$ is an open subset of $X . T$ is called compact if $\overline{T(X)}$ is a compact subset of $X$, where $\overline{T(X)}$ is a closure of $T(X)$ in $X$. Again $T$ is called totally bounded if for any bounded subset $S$

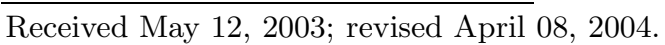

2000 Mathematics Subject Classification. 47H10.

Key words and phrases. Random fixed point theorem and random integralequation. 
of $X, T(S)$ is a totally bounded or precompact subset of $X$. Finally $T$ is called completely continuous if it is continuous and totally bounded. It is easy to see that every compact operator is totally bounded, but the converse may not be true. However, the two notions are equivalent on a bounded subset of $X$. Similarly a random operator $T: \Omega \times X \rightarrow X$ is continuous (resp. compact, totally bounded and completely continuous) if the operator $T(\omega)$ is continuous (resp. compact, totally bounded and completely continuous) on $X$ for each $\omega \in \Omega$.

An operator $T: X \rightarrow X$ is called $\mathcal{D}$-Lipschitzician if there exists a continuous and nondecreasing function $\phi: \mathbb{R}^{+} \rightarrow \mathbb{R}^{+}$such that

$$
\|T x-T y\| \leq \phi(\|x-y\|)
$$

for all $x, y \in X$, where $\phi(0)=0$. Sometimes we call the function $\phi$ to be a $\mathcal{D}$-function for $T$ on $X$. Similarly a random operator $T: \Omega \rightarrow X$ is called $\mathcal{D}$-Lipschitzician if there exists a continuous nondecreasing function $\phi: \Omega \times \mathbb{R}^{+} \rightarrow \mathbb{R}^{+}$such that for each $\omega \in \Omega$

$$
\|T(\omega) x-T(\omega) y\| \leq \phi_{\omega}(\|x-y\|)
$$

for all $x, y \in X$ where $\phi_{\omega}(r)=\phi(\omega, r)$ with $\phi(\omega, 0)=0$ for all $\omega \in \Omega$. If $\phi_{\omega}(r)=$ $\alpha(\omega) r, \alpha(\omega)$ being a positive real-valued function on $\Omega$, then $T(\omega)$ is called Lipschitzician with Lipschitz constant $\alpha(\omega)$. Further if $\alpha(\omega)<1$, for all $\omega \in \Omega$, then $T(\omega)$ is called contraction random operator with contraction constant $\alpha(\omega)$. Further if $\phi_{\omega}(r)<r, r>0$ for each $\omega \in \Omega$, where $\phi_{\omega}$ is not necessarily nondecreasing, then the random operator $T(\omega)$ is called nonlinear contraction on $X$. In the special case when the random parameter is absent, we obtain $T$ to be a deterministic nonlinear contraction on $X$. See Boyd and Wong [5].

A Kuratowskii measure of noncompactness $\alpha$ of a bounded set $S$ is defined to be a nonnegative real number $\alpha(S)$ given by

$$
\alpha(S)=\inf \left\{r>0: S=\bigcup_{i=1}^{n} S_{i}, \operatorname{diam}\left(S_{i}\right) \leq \epsilon, \forall i\right\} .
$$

A mapping $T: X \rightarrow X$ is called $\alpha$-condensing if for any bounded set $S$ of $X, f(S)$ is bounded and $\alpha(T(S))<\alpha(S)$ for $\alpha(S)>0$. The details of $\alpha$-condensing maps and fixed points appear in Banas and Goebel [27]. A random operator $T: \Omega \times X \rightarrow X$ is called $\alpha$-condensing if $\alpha(T(\omega)(S))<\alpha(S), \alpha(S)>0$ for each $\omega \in \Omega$. The random fixed point theory for $\alpha$-condensing random operators is initiated by Itoh [17] and developed subsequently by several authors. See Zeidler [27], Dhage [7] and the references therein.

In the present paper we shall prove the random versions of some fixed point theorems of the following type in Banach and ordered Banach spaces, and discuss some of their applications to random integral equations of mixed type under Carathéodory and monotonicity conditions.

Theorem 1.1. (Dhage [10]) Let $S$ be a non-empty closed and bounded subset of a Banach algebra $X$ and let $A, B, C: S \rightarrow X$ be three operators such that, 
(a) $A$ and $C$ are $\mathcal{D}$-Lipschitzicians with $\mathcal{D}$-functions $\phi$ and $\psi$ respectively,

(b) $B$ is completely continuous, and

(c) $A x B x+C x \in S$ for each $x \in S$.

Then the operator equations

$$
A x B x+C x=x
$$

has a solution in $S$, whenever $M \phi(r)+\psi(r)<r, r>0$ where

$$
M=\|B(S)\|=\sup \{\|B x\|: x \in S\} .
$$

\section{Topological Random Fixed Point Theory}

In this section we prove the random version of some hybrid fixed point theorems like Theorem 1.1 which combines a topological and a geometrical fixed point theorem via the method of measurable selectors. See Himmelberg [15] and Kuratowskii and RyllNardzewskii [20]. Before going to the main results of this paper, we state two useful Lemmas, the proof of which are implicit in Dhage [10], however we give here explicitly the details of proof.

Lemma 2.1. Let $S$ be a non-empty subset of a Banach algebra $X$ and let $A, B$, $C: S \rightarrow X$ be three operators satisfying

(a) $A$ and $C$ are $\mathcal{D}$-Lipschitzicians with $\mathcal{D}$-functions $\phi_{A}$ and $\phi_{C}$ respectively, and (b) $B$ is continuous and compact.

Then the mapping $T: S \rightarrow X$ defined by $T x=A x B x+C x$ is continuous.

Proof. Let $\left\{x_{n}\right\} \subset S$ be a sequence converging to a point $x \in S$. Then by the hypotheses (a)-(b),

$$
\begin{aligned}
\left\|T x_{n}-T x\right\| & =\left\|A x_{n} B x_{n}+C x_{n}-A x B x-C x\right\| \\
& \leq\left\|A x_{n} B x_{n}-A x B x\right\|+\left\|C x_{n}-C x\right\| \\
& \leq\left\|A x_{n}-A x\right\|\left\|B x_{n}\right\|+\|A x\|\left\|B x_{n}-B x\right\|+\left\|C x_{n}-C x\right\| \\
& \leq \phi_{A}\left(\left\|x_{n}-x\right\|\right)\|B x\|+\|A x\|\left\|B x_{n}-B_{x}\right\|+\phi_{C}\left(\left\|x_{n}-x\right\|\right) \\
& \rightarrow 0 \text { as } n \rightarrow \infty .
\end{aligned}
$$

Hence $T$ is continuous on $S$ and the proof of the lemma is complete.

Lemma 2.2. Let $S$ be a non-empty, bounded subset of Banach algebra $X$ and let $A$, $B, C: S \rightarrow X$ be three operators satisfying

(a) $A$ and $C$ are $\mathcal{D}$-Lipschitzicians with $\mathcal{D}$-functions $\phi_{A}$ and $\phi_{C}$ respectively, and

(b) $B$ is continuous and compact.

Then for any subset $G$ of $S$,

$$
\alpha(T(G)) \leq\|B(S)\| \phi_{A}(\alpha(G))+\phi_{C}(\alpha(G))
$$


where $T x=A x B x+C x, x \in S$ and $M=\|B(S)\|=\sup \{\|B x\|: x \in S\}$.

Proof. Let $x, y \in G$ be arbitrary. Then we have

$$
\begin{aligned}
\left\|T x_{n}-T y\right\| & =\|A x B x+C x-A y B y-C y\| \\
& \leq\|A x B x-A y B y\|+\|C x-C y\| \\
& \leq\|A x-A y\|\|B y\|+\|A y\|\|B x-B y\|+\|C x-C y\| \\
& \leq\|B(G)\| \phi_{A}(\|x-y\|)+\|B x\|+\|A y\|\|B x-B y\|+\phi_{C}(\|x-y\|) .
\end{aligned}
$$

Now for any $x_{0} \in G$,

$$
\begin{aligned}
\|A y\| & \leq\left\|A x_{0}\right\|+\left\|A x_{0}-A y\right\| \\
& \leq\left\|A x_{0}\right\|+\phi_{A}\left(\left\|x_{0}-y\right\|\right) \\
& \leq\left\|A x_{0}\right\|+\phi_{A}(\operatorname{diam} G) \\
& \leq\left\|A x_{0}\right\|+\phi_{A}(\operatorname{diam} S) \quad(\text { i.e. } G \subset S) \\
& =\beta \\
& \leq \infty,
\end{aligned}
$$

for all $y \in S$. Substituting this estimate in the inequality (2.3)

$$
\|T x-T y\| \leq\|B(S)\| \phi_{A}(\|x-y\|)+\phi_{C}(\|x-y\|)+\beta\|B x-B y\| .
$$

Let $\varepsilon>0$ be given. Then there exist subsets $G_{1}, G_{2}, \ldots, G_{n}$ of $G$ such that $G=\cup_{i=1}^{n} G$ and diam $G_{i}<\alpha(G)+\epsilon$ for all $i=1, \ldots, n$. Since $B(S)$ is compact for any $\eta>0$, $\alpha(B(S))<\eta / \beta$ and there exist subsets $F_{1}, \ldots, F_{n}$ of $B(S)$ such that $B(S) \cup_{i=1}^{m} F_{i}$ or equivalently $G \subset \bigcup_{j=1}^{m} B^{-1}\left(F_{j}\right)$ with $\operatorname{diam}\left(F_{j}\right)<\eta / \beta$ for every $j=1, \ldots, m$. Similarly since $C(S)$ is compact for any $\theta>0, \alpha(C(S))<\theta$ and there exist subsets $H_{1}, H_{2}, \ldots, H_{l}$ of such that $C(S)=\bigcup_{k=1}^{l} H_{k}$ which implies that

$$
G \subset \bigcup_{k=1}^{l} H^{-1}\left(H_{k}\right) \text { with } \operatorname{diam} H_{k}<\theta \text { for all } k=1, \ldots, l .
$$

By Lemma 2.1, $T$ is continuous on $S$ and so $T(G)$ is bounded subset of $X$. Also we have

$$
T(G)=\bigcup_{i, j, k} T\left(G_{i} \cap B^{-1}\left(F_{j}\right) \cap C^{-1}\left(H_{k}\right)\right) .
$$

Since $\operatorname{diam}\left(G_{i}\right)<\alpha(G)+\epsilon$ for all $i=1, \ldots, n$ we have

$$
\operatorname{diam}\left(G_{i} \cap B^{-1}\left(F_{j}\right) \cap C^{-1}\left(H_{k}\right)\right)<\alpha(G)+\epsilon
$$

for all $i=1, \ldots, n, j=1, \ldots, m$ and $k=1, \ldots, l$. From (2.4) and (2.6) it follows that

$$
\begin{aligned}
& \operatorname{diam}\left(T\left(G_{i} \cap B^{-1}\left(F_{j}\right) \cap C^{-1}\left(H_{k}\right)\right)\right. \\
< & \max \left\{M \phi_{A}(r)+\phi_{C}(r): r \in[\alpha(G), \alpha(G)+\epsilon]\right\}+\eta+\theta
\end{aligned}
$$


for all $i=1, \ldots, n, j=1, \ldots, m$ and $k=1, \ldots, l$. Now

$$
\alpha(T(G))=\max _{i, j, k} \operatorname{diam}\left(T\left(G_{i} \bigcap B_{-1}\left(F_{j}\right) \bigcap C^{-1}\left(H_{k}\right)\right)\right.
$$

Hence from (2.7) we obtain

$$
\alpha(T(G))<\max \left\{M \phi_{A}(r)+\phi_{C}(r): r \in[\alpha(G), \alpha(G)+\epsilon]\right\}+\eta+\theta
$$

Since $\epsilon, \eta$ and $\theta$ are arbitrary

$$
\alpha(T(G)) \leq M \phi_{A}(\alpha(G))+\phi_{C}(\alpha(G))
$$

for all $G \subset S$. This completes the proof.

Now we formulate the main results of this section.

Theorem 2.1. Assume that all the conditions of Theorem 2.1 hold, Then the set $\operatorname{Fix}(T)=\{x \in S \mid A x B x+C x=x\}$ is compact.

Proof. Define a mapping $T: S \rightarrow X$ by $T x=A x B x+C x$. Then by Theorem 1.1, $\phi \neq \operatorname{Fix}(T)=\{x \in S \mid A x B x+C x=x\}$. Moreover $\operatorname{Fix}(T)$ is a closed subset of $S$. To see this let $\left\{x_{n}\right\}$ be a sequence in $\operatorname{Fix}(T)$ converging to a point $x \in S$. We shall prove that $x \in \operatorname{Fix}(T)$. By Lemma $2.1 T$ is continuous, and so

$$
x=\lim _{n} x_{n}=\lim _{n} T\left(x_{n}\right)=T\left(\lim _{n} x_{n}\right)=T x .
$$

As a result $x \in \operatorname{Fix}(T)$ and so $\operatorname{Fix}(T)$ is a closed subset of $X$. If $\operatorname{Fix}(T)$ is not compact, then by Lemma 2.2 with $G=\operatorname{Fix}(T)$, we have

$$
\begin{aligned}
\alpha(\operatorname{Fix}(T)) & =\alpha(T(\operatorname{Fix}(T))) \\
& \leq M \phi_{A}(\alpha(\operatorname{Fix}(T)))+\phi_{C}(\phi(\operatorname{Fix}(T))) \\
& <\alpha(\operatorname{Fix}(T)),
\end{aligned}
$$

which is a contradiction. Therefore $\operatorname{Fix}(T)$ is compact. This completes the proof.

Theorem 2.2. Let $S$ be a closed convex and bounded subset of a separable Banach algebra $X$ and let $A, B, C: \Omega \times S \rightarrow X$ be three random operators satisfying for each $\omega \in \Omega$,

(a) $A(\omega)$ and $C(\omega)$ are $\mathcal{D}$-Lipschitizcians with $\mathcal{D}$-functions $\phi_{A}(\omega)$ and $\phi_{C}(\omega)$ respectively,

(b) $B(\omega)$ is completely continuous and

(c) $A(\omega) x B(\omega)+C(\omega) x \in S$, for $x \in S$.

Then the random equation

$$
A(\omega) x B(\omega) x+C(\omega) x=x
$$

has a random solution whenever $M(\omega) \phi_{A}(\omega)(r)+\phi_{C}(\omega)(r)<r, r>0$ for all $\omega \in \Omega$, where $M(\omega)=\|B(\omega)(S)\|=\sup \{\|B(\omega) x\|: x \in S\}$. 
Proof. Define an operator $T: \Omega \times S \rightarrow X$ by

$$
T(\omega) x=A(\omega) x B(\omega) x+C(\omega) x .
$$

Since $A(\omega), B(\omega), C(\omega)$ are random operators, $A(\omega) x, C(\omega) x$ and $C(\omega) x$ are $X$-valued random variables for each $x \in S$. As $X$ is a separable Banach algebra, the sum as well as the product of $X$-valued random variables is again $X$-valued random variable. As a result $T(\omega) x=A(\omega) x B(\omega) x+C(\omega) x$ is a $X$-valued random variable. Hence $T(\omega)$ is a random operator on $S$ which is continuous in view of Lemma 2.1. Define a multi valued $\operatorname{map} F: \Omega \rightarrow 2^{S}$ by

$$
F(\omega)=\{x \in S \mid A(\omega) x B(\omega) x+C(\omega) x=x\} .
$$

Clearly by Theorem 1.1, $F(\omega)$ is non-empty for each $\omega \in \Omega$. Moreover by Lemma 2.2, $F(\omega)$ is a compact subset of $S$ for each $\omega \in \Omega$. To finish, it is enough to prove that $F$ is measurable on $\Omega$. Let $C$ be a closed subset of $X$. Denote

$$
L(C)=\bigcap_{n=1}^{\infty} \bigcup_{x_{i} \in C_{n}}\left\{\omega \in \Omega \mid\left\|x_{i}-\left(A(\omega) x_{i} B(\omega) x_{i}+C(\omega) x_{i}\right)\right\|<2 / n\right\},
$$

where $C_{n}=\{x \in X \mid d(x, C)<1 / n\}$ and $d(x, C)=\inf \{d(x, c) \mid c \in C\}$. Obviously $L(C) \in \mathcal{A}$. We shall prove that $F^{-1}(C) \subset L(C)$. It is easy to prove that $F^{-1}(C) \subset L(C)$. Now proceeding with the arguments similar to that in the proof of Theorem 2.1 of Itoh [17] it is proved that $L(C) \subset F^{-1}(C)$. As a result $F^{-1}(C)=L(C)$. Hence $\mathrm{F}$ is measurable on $\Omega$. Since $F(\omega)$ is compact for each $\omega \in \Omega$, it has closed values on $\Omega$. Now an application of theorem of Kurtowskii and Ryll-Nardzewskii [20] yields that $F$ has a measurable selector that is, there is a measurable mapping $\xi: \Omega \rightarrow X$ such that $\xi(\omega) \in F(\omega)$ for each $\omega \in \Omega$. This further by definition of $F(\omega)$ which implies that

$$
\xi(\omega)=A(\omega) \xi(\omega) B(\omega) \xi(\omega)+C(\omega) \xi(\omega), \quad \omega \in \Omega .
$$

This completes the proof.

Corollary 2.1. Let $S$ be a closed convex and bounded subset of a separable Banach algebra $X$ and let $A, B, C: \Omega \times S \rightarrow X$ be three random operators satisfying for $\omega \in \Omega$,

(a) $A(\omega)$ and $B(\omega)$ are Lipschitzicians with the Lipschitz constants $\alpha(\omega)$ and $\beta(\omega)$ respectively,

(b) $B(\omega)$ is continuous and compact, and

(c) $A(\omega) x B(\omega) x+C(\omega) x \in S$ for each $x \in S$.

Then the random equation (2.10) has a random solution and the set of such random solutions is compact, whenever $\alpha(\omega) M(\omega)+\beta(\omega)<1$ for all $\omega \in \Omega$, where $M(\omega)=$ $\|B(\omega)(S)\|=\sup \{\|B(\omega) x\|: x \in S\}$.

In a recent paper [10], the present author has proved a companion of Leray-Schauder principle [14] involving three operators in a Banach algebra. A slight generalization of this nonlinear alternative is, 
Theorem 2.3. Let $U$ and $\bar{U}$ denote respectively the open bounded and closed bounded subsets of a Banach algebra $X$ containing the origin 0 and let $A, C: X \rightarrow X$ and $B: \bar{U} \rightarrow X$ be three operators satisfying

(a) $A$ and $C$ are $\mathcal{D}$-Lipschitzicians with $\mathcal{D}$-functions $\phi_{A}$ and $\phi_{C}$ respectively,

(b) $[(I-C) / A]^{-1}$ exist on $B(\bar{U}), I$ being the identity operator on $X$.

(c) $B$ is completely continuous and

(d) $M \phi_{A}(r)+\phi_{C}(r)<r, r>0$ where $M=\|B(\bar{U})\|=\sup \{\|B x\|: x \in \bar{U}\}$.

Then either

(i) the operator equation (1.6) has a solution in $\bar{U}$, or

(ii) there exist an $u \in \partial \bar{U}$ such that $\lambda A(u / \lambda) B u+\lambda C(u)=u$ for some $\lambda \in(0,1)$, where $\partial \bar{U}$ is the boundary of $\bar{U}$ in $X$.

Now we shall obtain a random version of Theorem 2.3 which is again a companion of the random version of Leray-Schauder principle.

Theorem 2.4. Let $U$ and $\bar{U}$ denote respectively the open bounded and closed bounded subsets of a separable Banach algebra $X$ containing the origin 0 and let $A, C: \Omega \times X \rightarrow X$ and $B: \Omega \times \bar{U} \rightarrow X$ be three random operators satisfying for each $\omega \in \Omega$,

(a) $A(\omega)$ and $C(\omega)$ are $\mathcal{D}$-Lipschitzicians with $\mathcal{D}$-functions $\phi_{A}(\omega)$ and $\phi_{C}(\omega)$ respectively,

(b) $[(I-C) / A]^{-1}(\omega)$ exists on $B(\omega)(\bar{U})$,

(c) $B(\omega)$ is completely continuous.

(d) $M(\omega) \phi_{A}(\omega)(r)+\phi_{C}(\omega)(r)<r, r>0$, where $M(\omega)=\|B(\omega) \bar{U}\|$, and

(e) there does not exist an $u \in \partial \bar{U}$ such that

$$
\lambda(\omega) A(\omega)(u / \lambda) B(\omega) u+\lambda(\omega) C(\omega) u=u(\omega)
$$

for any measurable function $\lambda: \Omega \rightarrow R^{+}$with $0<\lambda(\omega)<1$, where $\partial \bar{U}$ is a boundary of $U$.

Then the random equation (2.10) has a random solution in $U$.

Proof. The proof is similar to Theorem 2.2 and now here we invoke Theorem 2.3 instead of Theorem 1.1 in the proof.

An interesting Corollary to Theorem 2.3 in its applicable form is

Corollary 2.2. Let $B_{r}(0)$ and $\bar{B}_{r}(0)$ denote respectively the open and closed balls centered at the origin 0 of radius $r$ in a separable Banach algebra $X$. Let $A, C: \Omega \times X \rightarrow$ $X$ and $B: \Omega \times \bar{B}_{r}(0) \rightarrow X$ be three random operators satisfying for each $\omega \in \Omega$,

(a) $A(\omega)$ and $C(\omega)$ are Lipschitzicians with Lipschitz constants $\alpha(\omega)$ and $\beta(\omega)$, respectively,

(b) $A(\omega)$ is regular and $\left(\frac{I}{A}\right)(\omega)$ is one to one,

(c) $B(\omega)$ is completely continuous,

(d) $\alpha(\omega) M(\omega)+B(\omega)<1$ where $M(\omega)=\left\|B(\omega)\left(\bar{B}_{r}(0)\right)\right\|=\left\{\|B(\omega) x\|: x \in \overline{B_{r}(0)}\right\}$ and

(e) there does not exist an $u \in X$ with $\|u\|=r$ such that

$$
\lambda A(\omega)(u / \lambda) B(\omega) u+\lambda C(\omega) u=u(\omega) \quad \text { for any } \quad 0<\lambda<1 .
$$


Then the random equation (2.10) has a random solution in $\bar{B}_{r}(0)$.

Proof. Notice

$$
[(I-C) / A]^{-1}(\omega)=(I / A)^{-1}(I-C)^{-1}(\omega) .
$$

Since $C(\omega)$ is Lipschitzician with Lipschitz constant $\alpha(\omega)<1$, it is a contraction random operator on $X$, so by the random version of a Banach contraction principle yields that $(I-C)^{-1}(\omega)$ exists on $X$ and in particular on $B(\omega)\left(\bar{B}_{r}(0)\right)$. As $A(\omega)$ is regular, that is $A(\omega)$ maps $X$ into the set of all invertible elements in $X$ for each $\omega \in \Omega,(I / A)(\omega)$ is well defined. Further since $(I / A)(\omega)$ is one-to-one for each $\omega \in \Omega,(I / A)^{-1}(\omega)$ exists on $\Omega \times X$. As a result the random operator $[(I-C) / A]^{-1}(\omega)$ exists on $B(\omega)\left(\bar{B}_{r}(0)\right)$. Now the desired conclusion follows by an application of Theorem 2.4 by replacing $\bar{U}$ with $\bar{B}_{r}(0)$. This completes the proof.

When $C \equiv 0$ in Theorem 2.3 and Corollary 2.2 we obtain the following random versions of the fixed point theorems of Dhage [9] and Dhage and Regan [12]. See Dhage [13].

Theorem 2.5. Let $U$ and $\bar{U}$ denote respectively the open bounded and closed bounded subsets of a separable Banach algebra $X$ containing the origin 0 and let $A: \Omega \times X \rightarrow X$ and $B: \Omega \times \bar{U} \rightarrow X$ be three random operators satisfying for each $\omega \in \Omega$,

(a) $A(\omega)$ is a $\mathcal{D}$-Lipschitzician with $\mathcal{D}$-functions $\phi_{A}(\omega)$,

(b) $(I / A)^{-1}(\omega)$ exists on $B(\omega)(\bar{U})$,

(c) $B(\omega)$ is completely continuous.

(d) $M(\omega) \phi_{A}(\omega)(r)<r, r>0$, where $M(\omega)=\|B(\omega) \bar{U}\|$, and

(e) there does not exist an $u \in \partial \bar{U}$ such that

$$
\lambda(\omega) A(\omega)(u / \lambda) B(\omega) u=u(\omega)
$$

for any function measurable $\lambda: \Omega \rightarrow R^{+}$with $0<\lambda(\omega)<1$, where $\partial \bar{U}$ is a boundary of $U$.

Then the random equation (2.10) has a random solution in $U$.

Corollary 2.3. Let $B_{r}(0)$ and $\bar{B}_{r}(0)$ denote respectively the open and closed balls centered at the origin 0 of radius $r$ in a separable Banach algebra $X$. Let $A: \Omega \times X \rightarrow X$ and $B: \Omega \times \bar{B}_{r}(0) \rightarrow X$ be two random operators satisfying for each $\omega \in \Omega$,

(a) $A(\omega)$ is a Lipschitzician with Lipschitz constant $\alpha(\omega)$,

(b) $A(\omega)$ is regular and $\left(\frac{I}{A}\right)(\omega)$ is one to one,

(c) $B(\omega)$ is completely continuous,

(d) $\alpha(\omega) M(\omega)<1$ where $M(\omega)=\left\|B(\omega)\left(\bar{B}_{r}(0)\right)\right\|=\left\{\|B(\omega) x\|: x \in \bar{B}_{r}(0)\right\}$ and

(e) there does not exist an $u \in X$ with $\|u\|=r$ such that

$$
\lambda A(\omega)(u / \lambda) B(\omega) u=u(\omega) \quad \text { for any } \quad 0<\lambda<1 .
$$


Then the random equation (2.10) has a random solution in $\bar{B}_{r}(0)$.

\section{Algebraic Random Fixed Point Theory}

A non-empty closed subset $K$ of a Banach algebra $X$ is called a cone if (i) $K+K \subset K$, (ii) $\lambda K \subset K$ for all $\lambda \in \mathbb{R}^{+}$and (iii) $\{-K\} \cap\{K\}=0$, where 0 is a zero element of $X$. Further if $K$ satisfies (iv) $K \circ K \subset K$, where "०" is a multiplicative composition in $X$, then $K$ is called a positive cone in $X$.

We introduce an order relation $\leq$ in $X$ as follows.

Let $x, y \in X$. Then we say

$$
x \leq y \quad \text { if and only if } y-x \in K .
$$

Let $u, v \in X$ be such that $u \leq v$. Then by an order interval $[u, v]$ we mean a set in $X$ defined by

$$
[u, v]=\{x \in X \mid u \leq x \leq v\} .
$$

Let $a, b: \Omega \rightarrow X$. Then by $a \leq b$ we mean $a(\omega) \leq b(\omega)$ for all $\omega \in \Omega$ and we define an order interval $[a, b]$ by

$$
\begin{aligned}
{[a, b] } & =\{x \in X \mid a(\omega) \leq x \leq b(\omega) \forall \omega \in \Omega\} \\
& =\bigcap_{\omega \in \Omega}[a(\omega), b(\omega)] .
\end{aligned}
$$

An operator $T: X \rightarrow X$ is called nondecreasing if for any $x, y \in X, x \leq y$ implies $T x \leq T y$. Again the operator $T$ is called positive if range $(T) \subset K$. Similarly a random operator $T: \Omega \times X \rightarrow X$ is called nondecreasing (positive) if the operator $T(\omega)$ is nondecreasing (positive) for each $\omega \in \Omega$. The following lemma proved in Dhage [11] is useful in the sequel.

Lemma 3.1. Let $u_{1}, u_{2}, v_{1}, v_{2} \in K$ be such that $u_{1} \leq v_{1}$ and $u_{2} \leq v_{2}$. Then $u_{1} u_{2} \leq v_{1} v_{2}$.

A key theorem of this section is

Theorem 3.1. Let $X$ be a separable Banach algebra and let $a, b: \Omega \rightarrow X$ be two measurable functions such that $a \leq b$. Let $Q: \Omega \times[a, b] \rightarrow[a, b]$ be a continuous and nondecreasing random operator. Then $Q(\omega)$ has a minimal random fixed point $\xi *$ and a maximal random fixed point $\xi^{*}$ in $[a, b]$ if any one of the following conditions holds.

(a) $Q(\omega)$ is $\alpha$-condensing random operator and the cone $K$ in $X$ is normal.

(b) the cone $K$ is regular in $X$.

Moreover,

$$
\xi *(\omega)=\lim _{n} Q^{n}(\omega) a \quad \text { and } \quad \xi^{*}(\omega)=\lim _{n} Q^{n}(\omega) b .
$$

Our main result of this section is 
Theorem 3.2. Let $X$ be a separable Banach algebra and let $a, b: \Omega \rightarrow X$ be two measurable functions with $a \leq b$. Let $A, C: \Omega \times[a, b] \rightarrow K$ and $C: \Omega \times[a, b] \rightarrow X$ be three nondecreasing random operators satisfying for each $\omega \in \Omega$,

(a) $A(\omega)$ and $C(\omega)$ are $\mathcal{D}$-Lipschitzicians with $\mathcal{D}$-functions $\phi_{A}(\omega)$ and $\phi_{C}(\omega)$ respectively,

(b) $B(\omega)$ is completely continuous, and

(c) $A(\omega) x B(\omega) x+C(\omega) x \in[a, b]$ for each $x \in[a, b]$.

Further if the cone $K$ in $X$ is positive and normal, then the random equation (2.10) has a minimal random solution $\xi *$ and a maximal random solution $\xi^{*}$ in $[a, b]$ whenever $M(\omega) \phi_{A}(\omega)(r)+\phi_{C}(\omega)(r)<r$,for $r>0$ and for all $\omega \in \Omega$, where $M(\omega)=$ $\|B(\omega)([a, b])\|=\{\|B(\omega) x\|: x \in[a, b]\}$. Moreover

$$
\xi *(\omega)=\lim _{n} x_{n}(\omega) \quad \text { and } \quad \xi^{*}(\omega)=\lim _{n} y_{n}(\omega),
$$

where $x_{n+1}(\omega)=A(\omega) x_{n} B(\omega) x_{n}+C(\omega) x_{n}, n>0$ with $x_{0}(\omega)=a(\omega)$ and $y_{n+1}(\omega)=$ $A(\omega) y_{n} B(\omega) y_{n}+C(\omega) y_{n}, n \geq 0$ with $y_{0}(\omega)=b(\omega)$.

Proof. Define a mapping $Q(\omega): \Omega \times X \rightarrow X$ by

$$
Q(\omega) x=A(\omega) x B(\omega) x+C(\omega) x .
$$

Obviously by lemma 2.1, $Q(\omega)$ is a continuous random operator on $X$. By hypothesis (c), $Q$ defines a mapping $Q: \Omega \times[a, b] \rightarrow[a, b]$. Since the cone $K$ is normal, $[a, b]$ is a norm bounded subset of $X$. Let $\omega \in \Omega$ be fixed. Then for any subset $G$ of $[a, b]$, we have by Lemma 3.1,

$$
\begin{aligned}
\alpha(Q(\omega))(G) & \leq\|B(\omega)([a, b])\| \phi_{A}(\alpha(G))+\phi_{C}(\omega)(\alpha(G)) \\
& <\alpha(G),
\end{aligned}
$$

where $\alpha(G)>0$. Thus inequality (3.7) holds for every $\omega \in \Omega$ and so $Q(\omega)$ is $\alpha$-condensing random operator on $[a, b]$. Next we shall show that $Q(\omega)$ is a nondecreasing random operator on $[a, b]$. Let $x, y \in[a, b]$ be such that $x \leq y$. Since the cone $K$ is positive, by Lemma 3.1,

$$
\begin{aligned}
Q(\omega) x & =A(\omega) x B(\omega) x+C(\omega) x \\
& \leq A(\omega) y B(\omega) y+C(\omega) y \\
& =Q(\omega) y
\end{aligned}
$$

for all $\omega \in \Omega$. This shows that $Q$ is a nondecreasing random operator $[a, b]$. Now the desired conclusions follows by an application of Theorem 3.1.

Corollary 3.1. Let $X$ be a separable Banach algebra and let $A, B: \Omega \times X \rightarrow K$ and $C: \Omega \times X \rightarrow X$ be three nondecreasing random operators satisfying for $\omega \in \Omega$,

(a) $A(\omega)$ and $C(\omega)$ are Lipschitzicians with the Lipschitz constants $\alpha(\omega)$ and $\beta(\omega)$, respectively, 
(b) $B(\omega)$ is completely continuous and

(c) there exist two measurable functions $a, b: \Omega \rightarrow X$ such that

$$
a(\omega) \leq A(\omega) a B(\omega) a+C(\omega) a \text { and } A(\omega) B(\omega) b+C(\omega) b \leq b(\omega) .
$$

Further if the cone $K$ in $X$ is positive and normal, then random equation (2.10) has a minimal random solution $\xi *$ and a maximal random solution $\xi^{*}$ in $[a, b]$ whenever $\alpha(\omega) M(\omega)+\beta(\omega)<1$ for all $\omega \in \Omega$, where $M(\omega)=B(\omega)([a, b])=\{\|B(\omega) x\|: x \in[a, b]\}$. Moreover

$$
\xi *(\omega)=\lim _{n} x_{n}(\omega) \quad \text { and } \quad \xi^{*}(\omega)=\lim _{n} y_{n}(\omega),
$$

where the sequences $\left\{x_{n}(\omega)\right\}$ and $\left\{y_{n}(\omega)\right\}$ are defined by (3.5).

\section{Nonlinear Random Integral Equations}

Given a measurable space $(\Omega, \mathcal{A})$ and given a closed and bounded interval $J=$ $\left[t_{0}, t_{1}\right] \subset \mathbb{R}, \mathbb{R}$ a set of real numbers, consider the nonlinear functional random integral equations (in short FRIE) of mixed type,

$$
\begin{aligned}
x(t, \omega)= & h(t, x(\mu(t), \omega), \omega)+[f(t, x(\theta(t), \omega), \omega)] \\
& \times\left(q(t, \omega)+\int_{0}^{\sigma(t)} k(t, s, \omega) g(s, x(\eta(s), \omega), \omega) d s\right)
\end{aligned}
$$

for all $t \in J$ and $\omega \in \Omega$, where $q: J \times \Omega \rightarrow \mathbb{R}, k: J \times J \times \Omega \rightarrow \mathbb{R}, h, f, g: J \times \mathbb{R} \times \Omega \rightarrow \mathbb{R}$ and $\eta, \theta, \sigma, \eta: J \rightarrow J$.

The FRIE (4.1) is considerably general in the sense that it includes some deterministic as well as some indeterministic nonlinear integral equations that have been studied in the literature including those of Bharucha Reid [1], Dhage [10] and Dhage and Regan [12] as special cases. It is worthwhile to mention that the random version of Chandrasekhar's $H$-equation in the radioactive heat transfer is a particular case of FRIE (4.1). Therefore the study of the FRIE (4.1) has got importance for various aspects of its random solution. In this section we deal with only the existence theory for FRIE (4.1) via the abstract results developed in the previous section.

\subsection{Existence of solution}

Let $M(J, \mathbb{R}), B(J, \mathbb{R}), \operatorname{BM}(J, \mathbb{R})$ and $C(J, \mathbb{R})$ denote respectively the spaces of all measurable, bounded, bounded and measurable and continuous real-valued functions on $J$. Notice that $C(J, \mathbb{R}) \subset \operatorname{BM}(J, \mathbb{R}) \subset M(J, \mathbb{R})$.

We shall obtain the existence of the random solutions of the FRIE (4.1) in the space $\operatorname{BM}(J, \mathbb{R})$ under some suitable conditions. Define a norm $\|\cdot\|$ in $\operatorname{BM}(J, \mathbb{R})$ by

$$
\|x\|=\max _{t \in J}|x(t)| .
$$


Clearly $\operatorname{BM}(J, \mathbb{R})$ is a separable Banach algebra with this maximum norm. By $L^{1}(J, \mathbb{R})$ we denote the space of all Lebesgue integral real-valued functions on $J$ equipped with a norm $\|\cdot\|_{L^{1}}$ given by

$$
\|x\|_{L^{1}}=\int_{t_{0}}^{t_{1}}|x(s)| d s .
$$

We need the following definition in the sequel.

Definition 4.1. A mapping $\beta: J \times \mathbb{R} \times \Omega \rightarrow \mathbb{R}$ is said to satisfy a condition of $\omega$-Carathéodory or simply called $\omega$-Carathéodory if for each $\omega \in \Omega$,

(i) $t \mapsto \beta(t, x, \omega)$ is measurable for each $x \in \mathbb{R}$,

(ii) $x \mapsto \beta(t, x, \omega)$ is continuous almost everywhere for $t \in J$.

Further a $\omega$-Carathéodory function $\beta$ is called $L_{\omega}^{1}$-Carathéodory if

(iii) for each real number $r>0$ there exists a measurable function $h_{r}: \Omega \rightarrow L^{1}(J, \mathbb{R})$ such that $|\beta(t, x, \omega)| \leq h_{r}(t, \omega)$, a.e. $t \in J$ for all $x \in \mathbb{R}$ with $|x| \leq r$.

We consider the following hypotheses in the sequel.

$\left(A_{0}\right)$ The functions $\mu, \phi, \sigma, \eta: J \rightarrow J$ are continuous.

$\left(A_{1}\right)$ The function $q: \Omega \rightarrow C(J, \mathbb{R})$ is measurable.

$\left(A_{2}\right) k: \Omega \rightarrow C(J \times \mathbb{R}, \mathbb{R})$ is measurable.

$\left(A_{3}\right)$ The function $h: \Omega \rightarrow C(J \times \mathbb{R}, \mathbb{R})$ is measurable and there exists a function $\gamma: \Omega \rightarrow B(J, \mathbb{R})$ satisfying for each $\omega \in \Omega$,

$$
|h(t, x, \omega)-f(t, y, \omega)| \leq \gamma(t, \omega)|x-y|, \quad \text { a.e. } \quad t \in J
$$

for all $x, y \in \mathbb{R}$.

$\left(A_{4}\right) f$ defines a function $f: J \times \mathbb{R} \times \Omega \rightarrow \mathbb{R}-\{0\}$ and $\frac{x}{f(t, x, \omega)}=\frac{y}{f(t, y, \omega)}$ implies $x=y$ for all $t \in J$ and $\omega \in \Omega$.

$\left(A_{5}\right)$ The function $f: \Omega \rightarrow C(J \times \mathbb{R}, \mathbb{R})$ is measurable and there exists a function $\alpha: \Omega \rightarrow B(J, \mathbb{R})$ with bound $\|\alpha(\omega)\|$ satisfying for each $\omega \in \Omega$,

$$
|f(t, x, \omega)-f(t, y, \omega)| \leq \alpha(t, \omega)|x-y|, \quad \text { a.e. } \quad t \in J
$$

for all $x, y \in \mathbb{R}$.

$\left(A_{6}\right)$ The function $\omega \rightarrow g(t, x, \omega)$ is measurable $\forall t \in J$ and $x \in \mathbb{R}$.

$\left(A_{7}\right)$ The function $g(t, x, \omega)$ is $L_{\omega}^{1}$-Carathéodory.

$\left(A_{8}\right)$ exists a function $\phi: \Omega \rightarrow L^{1}(J, \mathbb{R})$ such that $\phi(t, \omega)>0$, a.e. $t \in J, \forall \omega \in \Omega$ and a continuous nondecreasing function $\psi:[0, \infty) \rightarrow(0, \infty)$ satisfying for each $\omega \in \Omega$,

$$
|g(t, x, \omega)| \leq \phi(t, \omega) \psi(|x|), \quad \text { a.e. } \quad t \in J
$$

for all $x \in \mathbb{R}$. 
Theorem 4.1. Suppose that hypothesis $\left(A_{0}\right)-\left(A_{7}\right)$ hold. Further if there exists a real number $r>0$ such that

$$
\left.\begin{array}{l}
\|\alpha(\omega)\|\left[\|q(\omega)\|+\|\phi(\omega)\|_{L^{1}} \psi(r)\right]+\|\gamma(\omega)\|<1 \\
r>\frac{H(\omega)+F(\omega)\left[\|q(\omega)+K(\omega)\| \phi(\omega) \|_{L^{1}} \psi(r)\right]}{1-\left[\|\alpha(\omega)\|\left\{\|q(\omega)\|+K(\omega)\|\phi(\omega)\|_{L^{1}} \psi(r)\right\}+\|\gamma(\omega)\|\right]}
\end{array}\right\}
$$

where $F(\omega)=\sup _{t \in J}|f(t, 0, \omega)|$, then the FRIE (4.1) has a random solution $\xi$ on $J$ with $\|\xi(\omega)\| \leq r$.

Proof. Let $X=B M(J, \mathbb{R})$ and consider an closed ball $\bar{B}_{r}(0)$ centered at the origin 0 in $X$ and of radius $r$, where $r$ satisfies the inequalities in (4.4). Define the operators $A$, $C: \Omega \times X \rightarrow X$ and $B: \Omega \times \bar{B}_{r}(0) \rightarrow X$ by

$$
\begin{aligned}
& A(\omega) x(t)=f(t, x(\theta(t), \omega), \omega), \quad t \in J \\
& B(\omega) x(t)=\int_{t_{0}}^{\sigma(t)} k(t, s, \omega) g(s, x(\eta(s), \omega), \omega) d s, \quad t \in J
\end{aligned}
$$

and

$$
C(\omega) x(t)=h(t, x(\mu(t), \omega), \omega), \quad t \in J
$$

Then the random integral equation (4.1) is equivalent to the random equation

$$
A(\omega) x(t) B(\omega) x(t)+C(\omega) x(t)=x(t, \omega), \quad t \in J .
$$

By hypothesis $\left(A_{3}\right)$ and $\left(A_{5}\right), A(\omega)$ and $C(\omega)$ are random operators on $X$. Since the functions $\omega \rightarrow k(t, s, \omega)$ and $\omega \rightarrow g(s, x, \omega)$ are measurable and $X$ is a separable Banach algebra, the function $\omega \rightarrow k(t, x, \omega) g(s, x, \omega)$ is measurable for all $t \in J$ and $x \in \mathbb{R}$. Again the integral

$$
\int_{0}^{\sigma(t)} k(t, s, \omega) g(s, x(\eta(s), \omega), \omega) d s,
$$

is the limit of a finite sum of measurable functions, so the function

$$
\omega \rightarrow \int_{0}^{\sigma(t)} k(t, s, \omega) g(s, x(\eta(s), \omega), \omega) d s,
$$

is measurable. Finally the sum of two measurable functions is again measurable, therefore $C(\omega)$ is a random operator on $X$. We shall show that the random operators $A(\omega), B(\omega)$ and $C(\omega)$ satisfy all the conditions of Corollary 3.1 .

Step I: We shall show that $A(\omega)$ and $C(\omega)$ are Lipschitzicians on $X$. Let $x, y \in X$. Then by hypothesis $\left(A_{3}\right)$ and $\left(A_{5}\right)$,

$$
\begin{aligned}
& \|A(\omega) x-A(\omega) y\| \\
& \quad=\sup _{t \in J}\{|f(t, x(\theta(t), \omega), \omega)-f(t, y(\theta(t), \omega), \omega)|\}
\end{aligned}
$$




$$
\begin{aligned}
& \left.\leq \sup _{t \in J}[\alpha(t, \omega) \mid x(\theta(t)), \omega)-y(\theta(t), \omega) \mid\right] \\
& \leq\left(\sup _{t \in J} \alpha(t, \omega)\right) \sup _{t \in J}|x(\theta(t), \omega)-y(\theta(t), \omega)| \\
& \leq\|\alpha(\omega)\|\|x(\omega)-y(\omega)\|,
\end{aligned}
$$

and so, $A(\omega)$ is a Lipschitzician with Lipschitz constant $\|\alpha(\omega)\|$. Similarly $C(\omega)$ is again Lipschitzician with Lipschitz constant $\|\gamma(\omega)\|$.

Step II : By hypothesis $\left(A_{4}\right)$,

$$
A(\omega) x(t)=f(t, x(\theta(t), \omega) \neq 0 \quad \forall t \in J
$$

and for all $\omega \in \Omega$, and so $A(\omega) x$ is a regular element of $X$ for all $x \in X$ and $\omega \in \Omega$. Further suppose that

$$
(I / A)(\omega) x=(I / A)(\omega) y \quad \text { for some } x, y \in X .
$$

Then $\frac{x(t, \omega)}{A(\omega) x(t)}=\frac{y(t, \omega)}{A(\omega) y(t)}$ for all $t \in J$. But in view of hypothesis $\left(A_{4}\right)$ which implies that $x(t, \omega)=y(t, \omega)$ for all $t \in J$ and $\omega \in \Omega$. Hence $x=y$ and as a result $\left(\frac{I}{A}\right)(\omega)$ is one-to-one on $X$.

Step III : Next we show that the random operator $B(\omega)$ is continuous and compact on $\overline{B_{r}(0)}$. Let $\left\{x_{n}\right\}$ be a sequence in $\overline{B_{r}(0)}$. Then $\left\|x_{n}\right\| \leq r$ for all $n \in \mathbb{N}$. Suppose that $x_{n} \rightarrow x$. By the dominated convergence theorem and the hypothesis $\left(A_{7}\right)$ we obtain

$$
\begin{aligned}
B(\omega) x_{n}(t) & =q(t, \omega)+\int_{0}^{\sigma(t)} k(t, s, \omega) g\left(s, x_{n}(\eta(s), \omega), \omega\right) d s \\
& \rightarrow q(t, \omega)+\int_{0}^{\sigma(t)} k(t, s, \omega) g\left(s, x_{n}(\eta(s), \omega), \omega\right) d s \\
& =B(\omega) x(t)
\end{aligned}
$$

for all $t \in J$, and so $B(\omega)$ is continuous random operator on $\bar{B}_{r}(0)$. To prove $B(\omega)$ is compact on $\bar{B}_{r}(0)$, it is enough to prove that the set $\left\{B(\omega) x_{n}: n \in \mathbb{N}\right\}$ is uniformly bounded and equi-continuous in $X$. From (4.8) it follows that

$$
\begin{aligned}
\left|B(\omega) x_{n}(t)\right| & \leq|q(t, \omega)|+\left|\int_{t_{0}}^{\sigma(t)} k(t, s, \omega)\right| g\left(s, x_{n}(\eta(s), \omega), \omega\right)|d s| \\
& \leq|q(t, \omega)|+\int_{t_{0}}^{t_{1}} K(\omega) h_{r}(s, \omega) d s \\
& =\|q(\omega)\|+K(\omega)\left\|h_{r}(\omega)\right\|_{L^{1}}
\end{aligned}
$$


for all $t \in J$ and $\omega \in \Omega$, where $h_{r}$ is a function given in Definition 4.1 (iii). From (4.7) it follows that $\left\{B(\omega) x_{n}: n \in \mathbb{N}\right\}$ is a uniformly bounded set in $X$. Further let $t, \tau \in J$. Then

$$
\begin{aligned}
& \left|B(\omega) x_{n}(t)-B(\omega) x_{n}(\tau)\right| \\
& \leq|q(t, \omega)-q(\tau, \omega)| \\
& +\mid \int_{t_{0}}^{\sigma(t)} k(t, s, \omega) g\left(s, x_{n}(\eta(s), \omega), \omega\right) d s \\
& \quad-\int_{t_{0}}^{\sigma(\tau)} k(\tau, s, \omega) g\left(s, x_{n}(\eta(s), \omega), \omega\right) d s \mid \\
& \leq|q(t, \omega)-q(\tau, \omega)| \\
& +\left|\int_{t_{0}}^{\sigma(t)}[k(t, s, \omega)-k(\tau, s, \omega)] g\left(s, x_{n}(\eta(s), \omega), \omega\right) d s\right| \\
& +\left|\int_{\sigma(\tau)}^{\sigma(t)} k(\tau, s, \omega) g\left(s, x_{n}(\eta(s), \omega), \omega\right) d s\right| \\
& \leq|q(t, \omega)-q(\tau, \omega)| \\
& +\left|\int_{t_{0}}^{\sigma(t)}\right| k(t, s, \omega)-k(\tau, s, \omega)|| g\left(s, x_{n}(\eta(s), \omega), \omega\right)|d s| \\
& +\left|\int_{\sigma(\tau)}^{\sigma(t)}\right| k(\tau, s, \omega)|| g\left(s, x_{n}(\eta(s), \omega), \omega\right)|d s| \\
& +\left|\int_{\sigma(\tau)}^{\sigma(t)}\right| k(\tau, s, \omega)\left|h_{r}(s) d s\right| \\
& \leq|q(t, \omega)-q(\tau, \omega)| \\
& +t_{t_{0}}^{\sigma(t)}|k(t, s, \omega)-k(\tau, s, \omega)| h_{r}(s) d s \mid
\end{aligned}
$$

where $p(t, \omega)=K(\omega) \int_{t_{0}}^{\sigma(t)} h_{r}(s, \omega) d s$.

Since the functions $t \rightarrow q(t, \omega), t \rightarrow k(t, s, \omega)$ are continuous on a compact interval $J$, they are uniformly continuous on $J$ for all $s \in J$ and $\omega \in \Omega$. Similarly since $\sigma: J \rightarrow J$ is continuous, the functions $t \rightarrow \sigma(t)$ is also uniformly continuous on $J$ for all $\omega \in \Omega$. Hence from the inequality (4.9), it follows that

$$
\left|B(\omega) x_{n}(t)-B(\omega) x_{n}(\tau)\right| \rightarrow 0 \quad \text { as } \quad t \rightarrow \tau .
$$


This shows that the set $\left\{B(\omega) x_{n}: n \in \mathbb{N}\right\}$ is equi-continuous set in $X$ for all $\omega \in \Omega$ Consequently the random operator $B(\omega)$ is continuous and compact on $\bar{B}_{r}(0)$.

Step IV : Here

$$
\begin{aligned}
M(\omega) & =\left\|B(\omega)\left(\bar{B}_{r}(0)\right)\right\| \\
& =\max \left\{|B(\omega) x|: x \in \bar{B}_{r}(0)\right\} .
\end{aligned}
$$

But

$$
\begin{aligned}
\|B(\omega) x\| & =\max _{t \in J}|B(\omega) x(t)| \\
& =\max _{t \in J}\left\{\left|q(t, \omega)+\int_{t_{0}}^{\sigma(t)} k(t, s, \omega) g(s, x(\eta(s), \omega), \omega)\right| d s\right\} \\
& \leq \max _{t \in J}|q(t, \omega)|+\max _{t \in J} \int_{t_{0}}^{\sigma(t)}|k(t, s, \omega) \| g(s, x(\eta(s), \omega), \omega)| d s \\
& \leq\|q(\omega)\|+K(\omega) \int_{t_{0}}^{\sigma(t)} \phi(s, \omega) \mid \psi(|x(\eta(s), \omega)|) d s \\
& \leq\|q(\omega)\|+K(\omega)\|\phi(\omega)\|_{L^{1}} \psi(r) .
\end{aligned}
$$

Therefore by (4.4)

$$
\alpha(\omega) M(\omega)+\beta(\omega) \leq\|\alpha(\omega)\|\left[q(\omega)\|+K(\omega)\| \phi(\omega) \|_{L^{1}} \psi(r)\right]+\|\gamma(\omega)\|<1
$$

for each $\omega \in \Omega$.

Step V : Suppose that the negation of hypothesis (e) of Corollary 3.1, holds, that is, there is an $u \in X$ with $\|u\|=r$ satisfying

$$
u(\omega)=\lambda A(u)\left(\frac{u}{\lambda}\right) B(\omega) u+\lambda C(\omega) u
$$

for some $0<\lambda<1$. Then we have

$$
u(t, \omega)=\lambda A(\omega)\left(\frac{u}{\lambda}\right)(t) B(\omega) u(t)+\lambda C(\omega) u(t) .
$$

Hence

$$
\begin{aligned}
|u(t, \omega)| \leq & |h(t, u(\mu(t), \omega), \omega)| \\
& +|f(t, u(\theta(t), \omega), \omega)|\left(|q(t, \omega)|+\int_{t_{0}}^{\sigma(t)} \mid k(t, s, \omega) g(s, u(\eta(s), \omega) \mid d s)\right. \\
\leq & |h(t, u(\mu(t), \omega), \omega)-h(t, 0, \omega)|+h(t, 0, \omega) \mid \\
& +[|f(t, u(\phi(t), \omega), \omega)-f(t, 0, \omega)|+|f(t, 0, \omega)|] \\
& \times\left[|q(t, \omega)|+\int_{t_{0}}^{\sigma(t)}|k(t, s, \omega)||g(s, u(\eta(s), \omega), \omega)| d s\right]
\end{aligned}
$$




$$
\begin{aligned}
\leq & \gamma(t, \omega)|u(\mu(t), \omega)|+H(\omega) \\
& +\alpha(t, \omega)|u(\theta(t), \omega)|\left(\|q(\omega)\|+K(\omega) \int_{t_{0}}^{t_{1}} \phi(s, \omega) \psi(|u(\eta(s), \omega)|) d s\right) \\
& +F(\omega)\left(\|q(\omega)\|+K(\omega) \int_{t_{0}}^{t_{1}} \phi(s, \omega) \psi(|u(\eta(s), \omega)|) d s\right) \\
\leq & \|\gamma(\omega)\|\|u(\mu(t), \omega)\|+H(\omega) \\
& +\|\alpha(\omega)\|\|u(\omega)\|\left[\|q(\omega)\|+K(\omega)\|\phi(\omega)\|_{L^{1}} \psi(\|u(\omega)\|)\right] \\
& +F(\omega)\left[\|q(\omega)\|+K(\omega)\|\phi(\omega)\|_{L^{1}} \psi(\|u(\omega)\|)\right] .
\end{aligned}
$$

Taking maximum over $t$,

$$
\begin{aligned}
\|u(\omega)\| \leq & \|\gamma(\omega)\|\|u(\omega)\|+H(\omega) \\
& +\|\alpha(\omega)\|\|u(\omega)\|\left[\|q(\omega)\|+K(\omega)\|\phi(\omega)\|_{L^{1}} \psi(\|u(\omega)\|)\right] \\
& +F(\omega)\left[\|q(\omega)\|+K(\omega)\|\phi(\omega)\|_{L^{1}} \psi(\|u(\omega)\|)\right] \\
\leq & \frac{H(\omega)+F(\omega)\left[\|q(\omega)\|+K(\omega)\|\phi(\omega)\|_{L^{1}} \psi(\|u(\omega)\|)\right]}{1-\left[\|\alpha(\omega)\|\left\{\|q(\omega)\|+K(\omega)\|\phi(\omega)\|_{L^{1}} \psi(\|u(\omega)\|)\right\}+\|\gamma(\omega)\|\right]}
\end{aligned}
$$

On substituting $\|u(\omega)\|=r$ in the above inequality yields

$$
r \leq \frac{H(\omega)+F(\omega)\left[\|q(\omega)\|+K(\omega)\|\phi(\omega)\|_{L^{1}} \psi(r)\right]}{1-\left[\|\alpha(\omega)\|\left\{\|q(\omega)\|+K(\omega)\|\phi(\omega)\|_{L^{1}} \psi(r)\right\}+\|\gamma(\omega)\|\right]}
$$

for all $\omega \in \Omega$. This contradicts to the second inequality in (4.4). Hence the hypothesis (e) of corollary 3.1 holds. Thus all the conditions of Corollary 3.1 are satisfied and hence an application of it yields that the random equation (4.7) and consequently the FRIE (4.1) has a random solution on J. This completes the proof.

\section{Existence of Extremal Solutions}

Now we prove the existence of maximal and minimal random solutions to FRIE (4.1) under certain additional monotonicity conditions.

Define an order relation $\leq$ in $B M(J, \mathbb{R})$ with the help of the cone $K$ in $B M(J, \mathbb{R})$ defined by

$$
K=\{x \in B M(J, \mathbb{R}) \mid x(t) \geq 0 \forall t \in J\} .
$$

Clearly the cone $K$ is positive and normal in $B M(J, \mathbb{R})$.

We need the following definitions in the sequel.

Definition 5.1. A random solution $x_{M}$ of the FRIE(4.1) is said to be maximal on $J$ if $\mathrm{x}$ is any other random solution, then $x(t, \omega) \leq x_{M}(t, \omega) \forall t \in J$ and $\omega \in \Omega$. Similarly a minimal random solution $x_{m}$ of the FRIE on $J$ is defined. 
Definition 5.2. A measurable function $u: \Omega \rightarrow B M(J, \mathbb{R})$ is called a lower random solution of the FRIE (4.1) on $J$ if for each $\omega \in \Omega$,

$$
\begin{aligned}
u(t) \leq & h(t, u(\mu(t), \omega), \omega) \\
& +[f(t, u(\theta(t), \omega), \omega)]\left(q(t, \omega)+\int_{t_{0}}^{\sigma(t)} k(t, s, \omega) g(s, u(\eta(s), \omega), \omega) d s\right)
\end{aligned}
$$

for all $t \in J$. Similarly a measurable function $v: \Omega \rightarrow B M(J, \mathbb{R})$ is called an upper random solution of the FRIE (4.1) if the above inequality is satisfied with a reverse sign.

We consider the following hypotheses in the sequel.

$\left(B_{0}\right) q, k$ and $f, q$ define the functions $q: J \times \Omega \rightarrow \mathbb{R}^{+}, k: J \times J \times \Omega \rightarrow \mathbb{R}^{+}$and $f, q: J \times \mathbb{R} \times \Omega \rightarrow \mathbb{R}^{+}$, where $\mathbb{R}^{+}$is the set of all nonnegative real numbers.

$\left(B_{1}\right)$ The function $g(t, x, \omega)$ is $L_{\omega}^{1}$-Carathéodory.

$\left(B_{2}\right)$ The functions $h(t, x, \omega) f(t, x, \omega)$ and $g(t, x, \omega)$ are nondecreasing in $x$ for all $t \in J$ and $\omega \in \Omega$.

$\left(B_{3}\right)$ The FRIE (4.1) has a lower random solution $u$ and an upper random solution $v$ on $J$ with $u \leq v$.

Remark 5.1. Suppose that the hypotheses $\left(B_{0}\right)-\left(B_{3}\right)$ hold and define a function $h_{c}: J \times \Omega \rightarrow \mathbb{R}$ by $h(t, \omega)=|g(t, u(t, \omega), \omega)|+|g(t, v(t, \omega), \omega)|, t \in J$ for all $\omega \in \Omega$. Then $\omega \rightarrow h_{c}(t, \omega)$ is measurable for all $t \in J$ and $t \rightarrow h_{c}(t, \omega)$ is Lebesgue integrable for each $\omega \in \Omega$. Further for each $\omega \in \Omega,|g(t, x(t, \omega), \omega)| \leq h_{c}(t, \omega)$, a.e. $t \in J$ for all $x \in[u, v]$.

Theorem 5.1. Assume that the hypotheses $\left(A_{0}\right)-\left(A_{3}\right),\left(A_{5}\right)-\left(A_{8}\right)$ and $\left(B_{1}\right)-\left(B_{3}\right)$ hold. Then the FRIE (4.1) admits a minimal random solution $\xi *$ and a maximal random solution $\xi^{*}$ on $J$ whenever

$$
\|\alpha(\omega)\|\left[\|q(\omega)\|+K(\omega)\left\|h_{c}(\omega)\right\|_{L^{1}}\right]+\|\gamma(\omega)\|<1
$$

for all $\omega \in \Omega$, where $h_{c}$ is given as in Remark 4.1 Moreover,

$$
\xi *(\omega)=\lim _{n} x_{n}(\omega) \quad \text { and } \quad \xi^{*}(\omega)=\lim _{n} y_{n}(\omega),
$$

where

$$
\begin{aligned}
x_{n+1}(t, \omega)= & h\left(t, x_{n}(\mu(t), \omega), \omega\right)+\left[f\left(t, x_{n}(\theta(t), \omega), \omega\right)\right] \\
& \times\left(q(t, \omega)+\int_{t_{0}}^{\sigma(t)} k(t, s, \omega) g\left(s, x_{n}(\eta(s), \omega), \omega\right) d s\right)
\end{aligned}
$$

for $n \geq 0$ with $x_{0}(t, \omega)=u(t, \omega)$; and

$$
\begin{aligned}
y_{n+1}(t, \omega)= & h\left(t, y_{n}(\mu(t), \omega), \omega\right)+\left[f\left(t, y_{n}(\theta(t), \omega), \omega\right)\right] \\
& \times\left(q(t, \omega)+\int_{t_{0}}^{\sigma(t)} k(t, s, \omega) g\left(s, y_{n}(\eta(s), \omega), \omega\right) d s\right)
\end{aligned}
$$


for $n \geq 0$ with $y_{0}(t, \omega)=v(t, \omega)$ for all $t \in J$ and $\omega \in \Omega$.

Proof. Let $X=B M(J, \mathbb{R})$. Define the operators $A(\omega), B(\omega), C(\omega): \Omega \times X \rightarrow X$ by (4.4) (4.5) and (4.6) respectively. We shall show that the operators $A(\omega), B(\omega)$ and $C(\omega)$ satisfy all the conditions of Corollary 3.1. It is shown as in the proof of the Theorem 4.1 that $A(\omega)$ and $C(\omega)$ are Lipschitzicians random operators and $B(\omega)$ is a completely continuous random operators on $[u, v]$. Here the Lipschitz constant of $A(\omega)$ is $\|\alpha(\omega)\|$ and Lipschitz constant of $C(\omega)$ is $\|\gamma(\omega)\|$. Again $M(\omega)=\|B(\omega)([a, b])\|$. Hypothesis $\left(B_{1}\right)$ implies that the random operators $A(\omega)$ and $B(\omega)$ are positive on $X$. We show that $A(\omega), B(\omega)$ and $C(\omega)$ nondecreasing on $X$. Let $x, y \in X$ be such that $x \leq y$. Then for each $\omega \in \Omega$,

$$
\begin{aligned}
& A(\omega) x(t)=f(t, x(t, \omega), \omega), \quad t \in J \\
& \leq f(t, y(\theta(t), \omega), \omega) \\
&=A(\omega) y(t), \\
& B(\omega) x(t)=q(t, \omega)+\int_{t_{0}}^{\sigma(t)} k(t, s, \omega) g(s, x(\eta(s), \omega), \omega) d s \\
& q(t, \omega)+\int_{t_{0}}^{\sigma(t)} k(t, s, \omega) g(s, x(\eta(s), \omega), \omega) d s \\
&=B(\omega) y(t)
\end{aligned}
$$

and

$$
\begin{aligned}
C(\omega) x(t) & =h(t, x(\mu(t), \omega), \omega) \\
& =h(t, x(\mu(t), \omega), \omega) \\
& =C(\omega) y(t)
\end{aligned}
$$

for all $t \in J$. This shows that $A(\omega), B(\omega), C(\omega)$ are nondecreasing random operators on $X$. Hypothesis $\left(B_{3}\right)$ implies that

$$
u(t, \omega) \leq A(\omega) u(t) B(\omega) u(t)+C(\omega) u(t)
$$

for all $t \in J$ and $\omega \in \Omega$ and

$$
v(t, \omega) \geq A(\omega) v(t) B(\omega) v(t)+C(\omega) v(t)
$$

for all $t \in J$ and $\omega \in \Omega$.

Finally

$$
\begin{aligned}
M(\omega) & =\|B(\omega)([u, v])\| \\
& =\sup \{\|B(\omega) x\|: x \in[u, v]\} \\
& =\max \left\{\max _{t \in J} q(t, \omega)+\max _{t \in J} \int_{t_{0}}^{\sigma(t)}|k(t, s, \omega) \| g(s, x(\eta(s), \omega), \omega)| d s\right\} \\
& \leq\|q(\omega)\|+K(\omega)\left\|h_{c}(\omega)\right\|_{L^{1}}
\end{aligned}
$$


and $h_{c}$ is a function given in remark 4.1. Therefore we have

$$
\begin{aligned}
\alpha(\omega) M(\omega)+\beta(\omega) & =\|\alpha(\omega)\|\left[\|q(\omega)\|+K(\omega)\left\|h_{c}(\omega)\right\|_{L^{1}}\right]+\|\gamma(\omega)\| \\
& <1 \quad \forall \omega \in \Omega .
\end{aligned}
$$

Hence an application of Corollary 3.1 yields that the FRIE has a minimal random solution $\xi^{*}$ in $[u, v]$ given by (4.13) and (4. 14) respectively. This completes the proof.

We note that hypothesis $\left(B_{3}\right)$ can be replaced with the following condition: $\left(B_{4}\right)$

There exist measurable functions $l_{1}, m_{1}: \Omega \rightarrow B M(J, \mathbb{R}), l_{2}, l_{3}, m_{2}, m_{3}: \Omega \rightarrow$ $B M\left(J, \mathbb{R}^{+}\right)$such that

$$
\begin{aligned}
& l_{1}(t, \omega) \leq h(t, x, \omega) \leq m_{1}(t, \omega) \\
& l_{2}(t, \omega) \leq f(t, x, \omega) \leq m_{2}(t, \omega)
\end{aligned}
$$

and

$$
l_{3}(t, \omega) \leq g(t, x, \omega) \leq m_{3}(t, \omega)
$$

for all $t \in J, x \in \mathbb{R}$ and $\omega \in \Omega$.

Theorem 5.2. Assume that the hypotheses $\left(A_{0}\right)-\left(A_{3}\right),\left(A_{4}\right)-\left(A_{8}\right),\left(B_{1}\right)-\left(B_{2}\right)$ and $\left(B_{4}\right)$ hold. Then the FRIE (4.1) has a minimal and a maximal random solution on $J$, whenever

$$
\|\alpha(\omega)\| K(\omega)\left\|h_{l}(\omega)\right\|_{L^{1}}+\|\gamma(\omega)\|<1
$$

for all $\omega \in \Omega$, where $h_{l}(t, \omega)=l_{3}(t, \omega)+m_{3}(t, \omega) \forall t \in J$ and $\omega \in \Omega$.

Proof. Define the function $u, v: \Omega \in B M(J, \mathbb{R})$ by

$$
u(t, \omega)=l_{1}(t, \omega)+l_{2}(t, \omega)\left(q(t, \omega)+\int_{t_{0}}^{\sigma(t)} k(t, s, \omega) l_{3}(s, \omega) d s\right)
$$

and

$$
v(t, \omega)=m_{1}(t, \omega)+m_{2}(t, \omega)\left(q(t, \omega)+\int_{t_{0}}^{\sigma(t)} k(t, s, \omega) m_{3}(s, \omega) d s\right)
$$

for all $t \in J$ and $\omega \in \Omega$. We show that the function $u$ and $v$ are respectively the lower and upper random solutions of the FRIE (4.1) on $J$. To see this, by hypothesis $\left(B_{4}\right)$,

$$
\begin{aligned}
& l_{1}(t, \omega) \leq h(t, x(\mu(t), \omega), \omega) \\
& 0 \leq l_{2}(t, \omega) \leq f(t, x(\theta(t), \omega), \omega)
\end{aligned}
$$

and

$$
0 \leq q(t, \omega)+\int_{t_{0}}^{\sigma(t)} k(t, s, \omega) l_{3}(s, \omega) d s \leq \int_{t_{0}}^{\sigma(t)} k(t, s, \omega) g(s, x(\eta(s), \omega), \omega) d s
$$


for all $t \in J, \omega \in \Omega$ and $x \in B M(J, \mathbb{R})$. Hence by Lemma 3.1,

$$
\begin{aligned}
u(t, \omega)= & h(t, u(\mu(t), \omega), \omega) \\
& +f(t, u(\theta(t), \omega), \omega)]\left(q(t, \omega)+\int_{t_{0}}^{\sigma(t)} k(t, s, \omega) g(s, x(\eta(s), \omega), \omega) d s\right)
\end{aligned}
$$

for all $t \in J$ and $\omega \in \Omega$ This shows that the function $u: \Omega \rightarrow B M(J, \mathbb{R})$ is a lower random solution of the FRIE (4.1) on $J$. Similarly it is proved that the function $v$ is an upper random solution of the FRIE (4.1) on $J$. The rest of the proof is similar to Theorem 4.2. We omit The details.

\section{Applications}

\subsection{Random initial value problems}

Given a measurable space $(\Omega, \mathcal{A})$ and given a closed and bounded interval $J=[0,1]$ in $\mathbb{R}$, consider the initial value problems of ordinary functional random differential equation (in short : FRDE) of neutral type,

$$
\left.\begin{array}{rl}
\left(\frac{x(t, \omega)-h(t, x(\mu(t), \omega), \omega)}{f(t, x(\theta(t), \omega), \omega)}\right)^{\prime} & =g(t, x(\eta(t), \omega), \omega), \quad \text { a.e. } \quad t \in J \\
x(0, \omega) & =q(\omega)
\end{array}\right\}
$$

for all $\omega \in \Omega$ where $q: \Omega \rightarrow \mathbb{R}$ is measurable $\mu, \theta, \eta: J \rightarrow J, f: J \times \mathbb{R} \times \Omega \rightarrow \mathbb{R}-\{0\}$ and $h: J \times \mathbb{R} \times \Omega \rightarrow \mathbb{R}$ are continuous and $g: J \times \mathbb{R} \times \Omega \rightarrow \mathbb{R}$. By the solution of the $F R D E(6.1)$ we mean a function $x \in A C(J, \mathbb{R})$ that satisfies $(6.1)$, where $A C(J, \mathbb{R})$ is a space of all absolutely continuous real-valued functions on $J$.

Notice that

$$
A C(J, \mathbb{R}) \subset C(J, \mathbb{R}) \subset B M(J, \mathbb{R}) .
$$

An existence theorem for FRDE (6.1) is

Theorem 6.1. Assume that the hypothesis $\left(A_{3}\right)-\left(A_{8}\right)$ hold. Further if there exists a real number $r>0$ such that

$$
\|\alpha(\omega)\|\left[\|q(\omega)\|+\|\phi(\omega)\|_{L^{1}} \psi(r)\right]+\|\gamma(\omega)\|<1
$$

and

$$
r \leq \frac{H(\omega)+F(\omega)\left[\|q(\omega)\|+K(\omega)\|\phi(\omega)\|_{L^{1}} \psi(r)\right]}{1-\|\alpha(\omega)\|\left[\|q(\omega)\|+K(\omega)\|\phi(\omega)\|_{L^{1}} \psi(r)\right]-\|\gamma(\omega)\|}
$$

for all $\omega \in \Omega$, then the $F R D E$ (6.1) has a random solution on $J$.

Proof. The FRDE (6.1) is equivalent to the FRIE

$$
\begin{aligned}
x(t, \omega)= & h(t, x(\mu(t), \omega), \omega) \\
& +[f(t, x(\theta(t), \omega), \omega)]\left(b(\omega)+\int_{0}^{t} g(s, x(\eta(s), \omega), \omega) d s\right)
\end{aligned}
$$


for all $t \in J$ and $\omega \in \Omega$, where $b(\omega)=\frac{q(\omega)-h(0, q(\omega), \omega)}{f(0, q(\omega), 0)}$.

Since $A C(J, \mathbb{R}) \subset B M(J, \mathbb{R})$, the desired conclusion follows by an application of Theorem 4.1 with $\sigma(t)=1, q(t, \omega)=b(\omega)$ and $k(t, s, \omega)=1$, for all $t, s \in J$ and $\omega \in \Omega$. The proof is complete.

To prove the existence of the extremal random solutions of the FRDE (6.1), we need the following definition.

Definition 6.1. A measurable function $u: \Omega \rightarrow A C(J, \mathbb{R})$ is called a lower random solution of the FRDE (6.1) if for each $\omega \in \Omega$.

$$
\begin{aligned}
\left(\frac{u(t, \omega)-k(t, u(\mu(t), \omega), \omega)}{f(t, x(\theta(t), \omega), \omega)}\right)^{\prime} \leq g(t, x(\eta(t), \omega), \omega), \quad \text { a.e. } \quad t \in J, \\
u(0, \omega) \leq q(\omega)
\end{aligned}
$$

for all $t \in J$. Similarly a measurable function $v: \Omega \rightarrow A C(J, \mathbb{R})$ is called an upper random solution of the FRDE (6.1) if the above inequalities are satisfied with a reverse sign.

Definition 6.2. A random solution $x_{M}$ of the FRDE (6.1) is called maximal if for any other random solution $x$, we have $x(t, \omega) \leq x_{M}(t, \omega)$ for all $t \in J$ and $\omega \in \Omega$. Similarly a minimal random solution $x_{m}$ of the FRDE (6.1) is defined.

Theorem 6.2. Assume that the hypotheses $\left(A_{5}\right)-\left(A_{8}\right)$ and $\left(B_{1}\right)-\left(B_{2}\right)$ hold. Suppose that FRDE (6.1) has a lower random solution $u$ and an upper random solution $v$ such that $u \leq v$. Further if

$$
\|\alpha(\omega)\|\left(|b(\omega)|+\left\|h_{c}(\omega)\right\|_{L^{1}}\right)+\|\gamma(\omega)\|<1,
$$

for all $\omega \in \Omega$, where $h_{c}$ is given in Remark 4.1, then the FRDE (6.1) has a minimal random solution $\xi_{*}$ and a maximal random solution $\xi^{*}$ in $[u, v]$. Moreover

$$
\xi_{*}(\omega)=\lim x_{n}(\omega) \quad \text { and } \quad \xi^{*}(\omega)=\lim y_{n}(\omega),
$$

where

$$
\begin{aligned}
x_{n+1}(t, \omega)= & h\left(t, x_{n}(\mu(t), \omega), \omega\right)+\left[f\left(t, x_{n}(\theta(t), \omega), \omega\right)\right] \\
& \times\left(b(\omega)+\int_{0}^{t} k(t, s, \omega) g\left(s, x_{n}(\eta(s), \omega), \omega\right) d s\right)
\end{aligned}
$$

for $n \geq 0$ with $x_{0}(t, \omega)=u(t, \omega)$; and

$$
\begin{aligned}
y_{n+1}(t, \omega)= & h\left(t, y_{n}(\mu(t), \omega), \omega\right)+\left[f\left(t, y_{n}(\theta(t), \omega), \omega\right)\right] \\
& \times\left(b(\omega)+\int_{0}^{t} k(t, s, \omega) g\left(s, y_{n}(\eta(s), \omega), \omega\right) d s\right)
\end{aligned}
$$


for $n \geq 0$ with $y_{0}(t, \omega)=v(t, \omega)$ for all $t \in J$ and $\omega \in \Omega$.

Proof. The proof is similar to Theorem 5.1 and now the conclusion follows by an application of Theorem 4.2.

\subsection{Integral equation in radiative heat transfer}

The deterministic Chandresekhar's $H$-equation in the radiative heat transfer is

$$
x(t)=1+x(t) \int_{0}^{t} t /(t+s) \psi(s) x(s) d s
$$

for all $t \in J=[0,1] \subset \mathbb{R}$, where $\psi: J \rightarrow \mathbb{R}$ is Lebesgue integrable. Attempts have been made to obtain the existence theorems for the integral equation (6.7) under suitable conditions on the function $\psi$ involved in it. The following existence result is noteworthy.

Theorem 6.3. (Chandresekhar [6]) If $\int_{0}^{t} \psi(s) d s \leq 1 / 2$, then the integral equation (6.7) is approximately strongly solvable.

We note that the process of radiative heat transfer involves the coefficient of heat transfer which may depend upon the medium of heat conduction. Hence the random version of the integral equation (6.7) is more appropriate to the needs of a specialist engaged in the accurate study of the underlined phenomenon. Below in the following section, we shall prove the existence theorem for the random version of integral equation (6.7) under suitable conditions.

\subsection{Random version of Chandresekhar's $H$-equation}

Given a measurable space $(\Omega, \mathcal{A})$ and given a closed and bounded interval $J=[0,1]$ in $\mathbb{R}$, consider the random integral equation

$$
x(t, \omega)=1+x(t, \omega) \int_{0}^{t} t /(t+s) \psi(s, \omega) x(s, \omega) d s
$$

for all $t \in J$ and $\omega \in \Omega$, where $\psi: \Omega \rightarrow L^{1}(J, \mathbb{R})$ is measurable. We shall obtain an existence result for the $R I E(6.8)$ in the space $B M(J, \mathbb{R})$ of all bounded and measurable real valued functions on $J$.

Theorem 6.4. Suppose that there exists a real number $r>1$ satisfying

$$
\|\psi(\omega)\|_{L^{1}} \leq \frac{1}{r}
$$

for all $\omega \in \Omega$. Then the RIE (6.8) has a random solution on $J$.

Proof. Let $X=B M(J, \mathbb{R})$ which is clearly a Banach algebra with respect to a norm

$$
\|x\|_{B M}=\max _{t \in J}\|x(t)\|
$$


and the product $(x . y)(t)=x(t) x(t)$, for every $t \in J$. Consider a closed ball $\bar{B}_{r}(0)$ in $X$ centered at origin 0 of radius $r$, where $r$ satisfies the inequality (6.9). Define the operators $A, B, C: \Omega \times \overline{B_{r}(0)} \rightarrow X$ by

$$
\begin{aligned}
& A(\omega) x(t)=x(t, \omega), \quad t \in J, \\
& B(\omega) x(t)=\int_{0}^{1} \frac{t}{(t+s)} \psi(s, \omega) x(t, \omega) d s, \quad t \in J
\end{aligned}
$$

and

$$
C(\omega) x(t)=1, \quad t \in J
$$

for all $\omega \in \Omega$.

Without loss of generality we may assume that $\|\psi(\omega)\|_{L^{1}} \leq \frac{r-1}{r^{2}}$, since we have that $\frac{r-1}{r^{2}}<\frac{1}{r}$. Then it is easy to verify that $A(\omega), B(\omega), C(\omega)$ are random operators and satisfy all the conditions of Corollary 2.1 and hence an application of it yields that $R I E$ (6.8) has a random solution on $J$.

Remark 6.1. The special case when $r=2$ in (6.9), we obtain an existence result for the integral equation (6.7) similar to the Theorem 6.3 with a simple but different method from that given in Chandresekhar [6].

\section{References}

[1] A. T. Bharucha Reid, Random Integral Equations, Academic Press, New York, 1972.

[2] A. T. Bharucha Reid, Fixed point theorems in probabilistic analysis, Bull. Amer. Math. Soc. 82 (1996), 611-645.

[3] A. T. Bharucha Reid, On the theory of random equations, Proc. Symp. Appl . Math. 16 ${ }^{\text {th }}$ (1963), 40-69, Amer. Math. Soc., Providence, rhode Island, 1964.

[4] A. T. Bharucha Reid, On random operator equations in Banach spaces, Bull. Acad. Polon. Sci. Ser. Sci. Math. Astronom. Phys. 7 (1959), 561-564.

[5] D. W. Boyd and J. S. W. Wong, On nonlinear contractions, Proc. Amer. Math. Soc. 20 (1969), 456-464.

[6] S. Chandrasekhar, Radiative Heat Transfer, Dover, New York, 1980.

[7] B. C. Dhage, On $\alpha$-condensing mappings in Banach algebras, The Math. Student 64 (1994), 146-152.

[8] B. C. Dhage, On some nonlinear alternatives of Leray-Schauder type with applications to functional integral equations, (submitted).

[9] B. C. Dhage, On some variants of Schauder's fixed point principle and applications to nonlinear integral equations, J. Math. Phy. Soc. 25 (1988), 603-611.

[10] B. C. Dhage, On existence theorem for nonlinear integral equations in Banach algebras via fixed point technique, East Asian Math. J. 17 (2001), 33-45.

[11] B. C. Dhage, Monotone iterative technique for Canrathéodory theory of nonlinear functional random integral equations, Tamkang J. Math. 33 (2002), 341-351.

[12] B. C. Dhage and D. O Regan, A fixed point theorem in Banach algebras with applications to functional integral equations, Functional Differential Equations 7 (2000), 259-267. 
[13] B. C. Dhage, Random fixed point theorems in Banach algebras with applications to random integral equations, Tamkang J. Math. 34 (2003), 29-43.

[14] J. Dugundji and A. Granas, Fixed Point Theory, Monographie Mathematyczne Vol. 1 PNW Warsaw, 1982.

[15] C. J. Himmelbeg, Measurable relations, Fund. Math. 87 (1975), 53-72.

[16] P. Hans, Random fixed point theorems, Transactions of the first prague Conference on Information Theory, Statistical Decision Functions, Random Process, pp. 105-125, 1957.

[17] S. Itoh, Random fixed point theorems with application to random differential equations in Banach spaces, J. Math. Anal. Appl. 67 (1979), 261-273.

[18] M. A. Kransoselskii, Topological Methods in the Theory of Nonlinear Integral Equations, Pergamon Press, 1964.

[19] K. Kuratowskii, Topology Vol. I, Hafner, New York, 1966.

[20] A general theorem on selectors, Bull. Acad. Polons, Scio. Ser. Math. Sci. Astr. Phys. 13 (1965), 397-403.

[21] M. A. Krasnoselskil, Topological Methods in the theory of Nonlinear Integral Equations, Translated from the Russian. Macmillan, New York, 1964.

[22] N. S. Papageorgiou, On the measurable selection approach in random differential inclusions, fixed point theory and optimization, J. Math. Phy. Sci. 24 (1990), 331-345.

[23] B. L. S. Prakas Rao, Stochastic integral equations of mixed type II, J. Math. Phy. Sci. 7 (1973), 245-260.

[24] N. S. Papageorgiu, Random fixed point theorems and differential inclusions, Intern. J. Math. \& Math. Sci. 11 (1988), 551-560.

[25] A. Spacek, Zufallige Gleichungen, Czechaslovak Math. J. 5 (1955), 462-466.

[26] T. T. Soong, Random Differential Equations in Sciences and Engineering, Academic Press, New York, 1973.

[27] E. Zeidler, Nonlinear Functional Analysis and Its Applications: Part-I, Springer Verlag, 1985.

Kasubai, Gurukul Colony, Ahmedpur-413 515, Dist Latur, Maharashtra, India.

E-mail:bcd20012001@yahoo.co.in 